\title{
The structure and kinematics of the $\mathrm{HH} 444$ photoionized jet in the $\sigma$ Orionis region
}

\author{
L. López-Martín ${ }^{1}$, A. C. Raga ${ }^{1}$, J. A. López ${ }^{2}$, and J. Meaburn ${ }^{3}$ \\ 1 Instituto de Astronomía, UNAM, Circuito de la Investigación Científica, 04510 Cd. Universitaria, D. F., Mexico \\ e-mail: luislm,raga@astroscu.unam.mx \\ 2 Instituto de Astronomía, UNAM, Km 103 Carretera Tijuana-Ensenada, 22860 Ensenada, B. C., Mexico \\ e-mail: jal@astrosen.unam.mx \\ 3 Astronomy Department, University of Manchester, Jodrell Bank, Macclesfield, Cheshire SK11 9DL, UK
}

Received 29 November 2000 / Accepted 2 March 2001

\begin{abstract}
HH} 444$ is a stellar jet which is immersed in the H II region photoionized by $\sigma$ Orionis. Spatially resolved, high spectral resolution observations of the $\mathrm{H} \alpha$ and [N II] $\lambda \lambda 6548,6584$ lines have been obtained for this object. These observations reveal for the first time the detailed velocity structure of the stellar jet and its associated bow-shock. The line profiles are modeled with gasdynamic numerical simulations, using a 3D code that also includes the transfer of the ionizing radiation from an external source (i.e., $\sigma$ Orionis for the case of HH 444). In order to reproduce the observed characteristics of the line profiles, we find that an axially peaked initial velocity profile for the jet and a non-zero initial opening angle for the flow with a time-variable ejection velocity is required. With these three ingredients we can reproduce the observed "acceleration" of the material along the jet beam, the rapid $\mathrm{H} \alpha$ intensity drop for increasing distances from the source, and the knotty structure of the jet, respectively.
\end{abstract}

Key words. ISM: jets and outflows - hydrodynamics - shock waves - ISM: individual: HH 444

\section{Introduction}

The discovery of jets with Herbig-Haro type characteristics in the $\sigma$ Orionis region (Reipurth et al. 1998) has prompted investigations on the photoionization of a neutral jet by an external source (Raga et al. 2000). Similar cases of $\mathrm{HH}$ jets immersed in an $\mathrm{H}$ II environment have also been found in the Orion nebula (Bally et al. 2000; Bally \& Reipurth 2001).

In typical $\mathrm{HH}$ jets the emission only comes from the regions of the beam which are excited by shocks whereas the beams of externally photoionized jets are composed mainly of emitting material. This qualitative difference makes the photoionized jets especially interesting, since in these objects we can directly observe most of the material that participates in the outflow. For this reason, studies of the interplay between the jet dynamics and an external photoionization source are a subject of considerable interest.

Raga et al. (2000) carried out analytic and numerical studies of an initially neutral jet which emerges from the circumstellar region into an environment that is being photoionized by an external source. For objects such as

Send offprint requests to: L. López-Martín, e-mail: luislm@astroscu.unam.mx those in the $\sigma$ Orionis region the ionization front simply "unveils" the jet beam, and has no important dynamic effects (as the front is a highly supersonic, R-type front).

In order to further test the physical conditions of photoionized jets, in this paper we present long-slit echelle observations of the HH 444 jet, which was studied with imaging and low resolution spectroscopy by Reipurth et al. (1998). We have obtained spatially resolved, high spectral resolution observations of the $\mathrm{H} \alpha$ and $[\mathrm{N}$ II] $\lambda \lambda 6548,6584$ lines, which are described in Sect. 2. We then use these observations (together with the previous observational results of Reipurth et al. 1998) to derive the parameters of the HH 444 outflow (Sect. 3). Finally, we use these parameters to compute gasdynamic models for this outflow, which we compare directly with the observations (Sect. 4).

\section{The observations}

High resolution, long-slit spectra of the HH 444 outflow were obtained with the Manchester Echelle Spectrograph (MES; Meaburn et al. 1984), at the $\mathrm{f} / 7.9$ focus of the $2.1 \mathrm{~m}$ telescope of the OAN (the Observatorio Astronómico Nacional, at San Pedro Mártir). This spectrometer has no cross-dispersion. The detector was a Tektronix CCD with $1024 \times 1024,24 \mu \mathrm{m}(0.3 \mathrm{arcsec})$ square pixels. A $2 \times 2$ pixel binning was employed in both the spatial and 


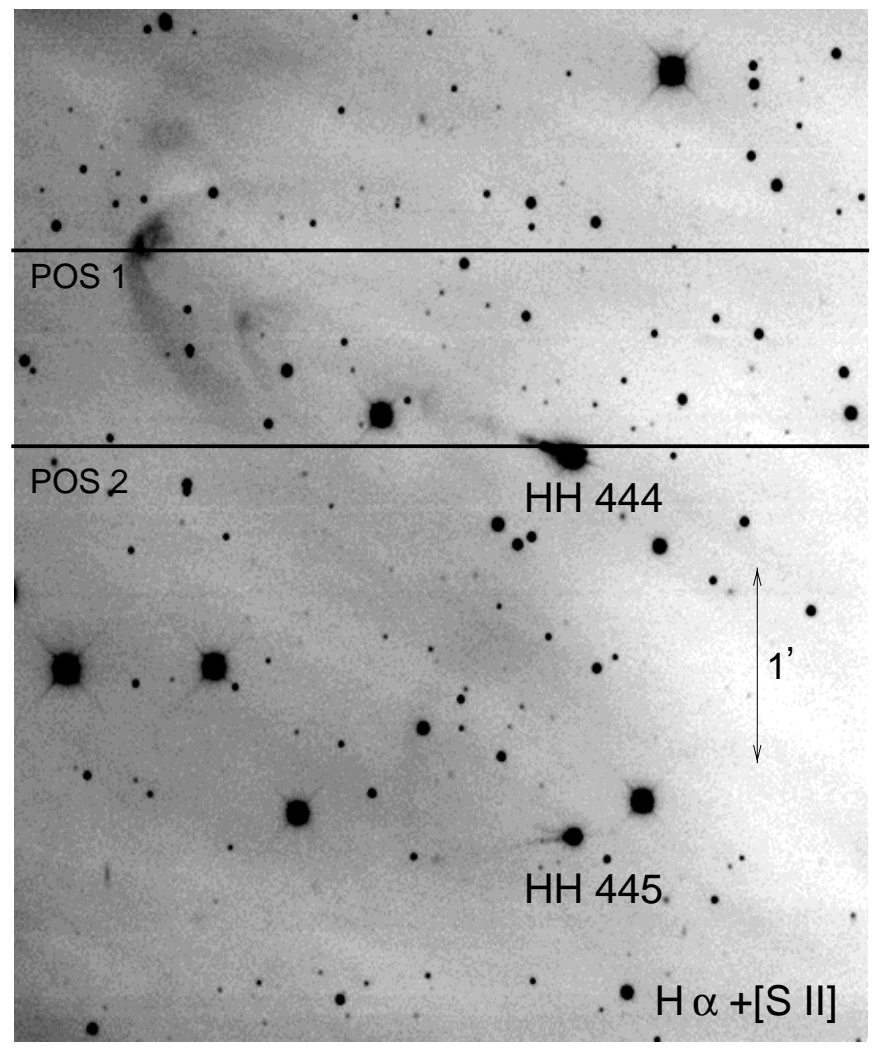

Fig. 1. Image of the region with the HH 444 and HH 445 jets in the light of $\mathrm{H} \alpha+[\mathrm{S} \mathrm{II}]$ (Reipurth et al. 1998). The slit positions (POS 1 and POS 2) are marked with the two horizontal lines. North is to the top

spectral dimensions. Consequently 512 increments, each of 0.60 arcsec long, gave a total projected slit length of 5.12 arcmin on the sky. The slit was 150 microns wide (= $12 \mathrm{~km} \mathrm{~s}^{-1}$ and 1.9 arcsec). For the present observations, a filter of $90 \AA$ bandwidth was used to isolate the $\mathrm{H} \alpha$ and $[\mathrm{N} \mathrm{II}] \lambda 6584$ nebular emission lines. The spectra were calibrated to $\pm 1 \mathrm{~km} \mathrm{~s}^{-1}$ accuracy using a Th-Ar arc lamp.

Observations with two east-west slit positions were obtained, one covering the base of the jet, and the other one cutting through the main bow shock. The locations of the two slit positions are shown in Fig. 1 on the $\mathrm{H} \alpha+[\mathrm{S} \mathrm{II}]$ image of Reipurth et al. (1998). These slit positions are particularly well known because a plane mirror can be inserted into the beam, before the echelle grating, permitting an image to be taken of the slit against that of the field being observed. The slit labeled as POS 2 in Fig. 1 was placed 4 arcsec north of the star. Photospheric continuum was detected in the spectrum cutting across the base of the $\mathrm{HH} 444$ jet, indicating that the emission from the star was not completely excluded. This continuum of the star has been extracted from the spectra of the base of the jet.

Exposures of $1800 \mathrm{~s}$ were taken for each slit position. The "seeing" varied between 1 and 1.5 arcsec during the observations.

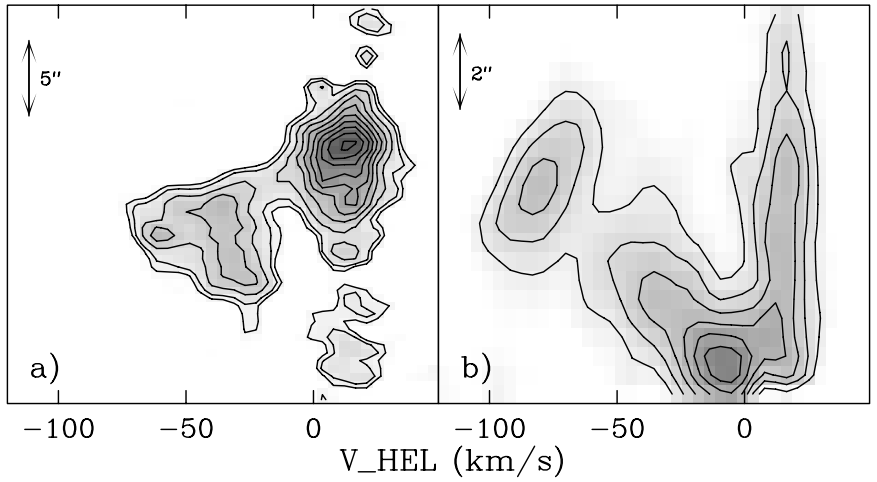

Fig. 2. Contour map, (with linearly spaced contours) of the $\mathrm{H} \alpha$ position-velocity map, a) for the POS1 slit (see Fig. 1) over the bow-shock of HH 444. The nebular emission from the $\mathrm{H}$ II region has been substracted, b) for the POS2 slit (see Fig. 1) over the base of $\mathrm{HH} 444$. The nebular emission from the H II region and stellar continuum have been substracted

The $\mathrm{H} \alpha$ line profiles of the bow shock of the HH 444 jet are shown in Fig. 2. Angular scale marks in these figures inicate the portions of the bow shock and the jet beam intersected by the slit. We see that the line profiles have two peaks, which form two ridges in the position-velocity diagrams. The line emission is detected over a $\sim 15^{\prime \prime}$ region, and a total radial velocity range of $\approx 100 \mathrm{~km} \mathrm{~s}^{-1}$ is observed, with the more negative radial velocities corresponding to the central region of the bow shock.

The corresponding line profiles for the base of the jet are also shown in Fig. 2. These position-velocity diagrams reveal a clear velocity gradient, with low radial velocities close to the star and increasingly negative radial velocities at larger distances, peaking at $\sim-100 \mathrm{~km} \mathrm{~s}^{-1}$ at $\sim 10^{\prime \prime}$ to the $\mathrm{E}$ of the star. The $\mathrm{H} \alpha$ and [N II] $\lambda 6584$ intensities first decrease with increasing distances from the source, and then show a rise to a strong peak at $\sim 10^{\prime \prime}$, where a well defined knot is observed along the jet. At larger distances from the source, the jet emission no longer falls along our east-west orientated spectrograph slit (see Fig. 2).

This last point is an important limitation of our observations. The clear misalignment between the E-W spectrograph slit and the direction of the jet (see Fig. 1) not only produces a cutoff in the detected jet emission at angular distances greater than $\sim 15^{\prime \prime}$ from the source, but could also be producing a cutoff in the emission detected from the base of the jet (which already might lie outside our spectrograph slit). However, in our spectra we do see a spatially unresolved stellar continuum, so that the point spread function of the central star does appear to fall at least partially within the spectrograph slit. Therefore, we do not expect that a substantial part of the emission from the base of the HH 444 jet is falling outside the spectrograph slit, so that the position-velocity diagrams that we have obtained for this region are more or less reliable. 


\subsection{The parameters for the $\mathrm{HH} 444$ jet}

The physical parameters of HH 444 have been estimated by Reipurth et al. (1998). Following these authors, we assume a distance to $\sigma$ Orionis of 400 pc. The projected separation between the source of the HH 444 jet (V 510 Ori) and $\sigma$ Orionis then has a value of $\approx 1.73 \mathrm{pc}$.

The ionizing source ( $\sigma$ Orionis) actually is a multiple star with an 09.5 star as the brightest component. We assume for this star an effective temperature $T_{\text {eff }}=$ $31000 \mathrm{~K}$ and a total production rate of ionizing photons $S_{\star}=10^{48} \mathrm{~s}^{-1}$.

Reipurth et al. (1998) obtain an electron density of $\approx 200 \mathrm{~cm}^{-3}$ for the jet beam from the red [S II] line ratio. Following Raga et al. (2000), we assume a somewhat higher $n_{\mathrm{j}}=500 \mathrm{~cm}^{-3}$ density for the base of the jet. The observed angular diameter of this jet is of $\approx 1^{\prime \prime}$, corresponding to a $r_{\mathrm{j}}=310^{15} \mathrm{~cm}$ jet radius.

From the spectrum of the bow-shock of HH 444 (see Fig. 2) it is possible to estimate the maximum and minumum radial velocity and with them we calculate the orientation angle $\phi$ between the outflow axis and the plane of the sky (Hartigan et al. 1987), giving a value of $\approx 40^{\circ}$ assuming that the material is outflowing into a stationary medium. Using this orientation angle and the maximum (i.e., more negative) observed radial velocities, we obtain a $v_{\mathrm{j}}=125 \mathrm{~km} \mathrm{~s}^{-1}$ jet velocity for $\mathrm{HH} 444$.

\section{A model for $\mathrm{HH} 444$}

We have computed numerical models of an initially neutral jet emerging into an ionizing radiation field with the new "yguazú" code, which is described in detail by Raga et al. (1999). This 3D code integrates the gas dynamic equations, a rate equation for neutral hydrogen, and the radiative transfer of the ionizing radiation (at the Lyman limit). The radiative cooling is computed with a simple, parametrized approximation to the real, non-equilibrium cooling.

The models have been computed in a uniform, Cartesian grid of $N_{x} \times N_{y} \times N_{z}=40 \times 40 \times 100$ grid points. The ionizing photon source is placed at a position $\left(x_{\mathrm{s}}, y_{\mathrm{s}}, z_{\mathrm{s}}\right)=(-1.73 \mathrm{pc}, 0,0)$. The jet is assumed to be initially neutral, and the surrounding, undisturbed environment is fully ionized. For the jet beam we impose an initial, top-hat density and temperature profile with $n_{\mathrm{j}}=500 \mathrm{~cm}^{-3}$ and $T_{\mathrm{j}}=1000 \mathrm{~K}$, and for the environment we assume a density $n_{\mathrm{env}}=10 \mathrm{~cm}^{-3}$ and $T_{\mathrm{env}}=10000 \mathrm{~K}$.

We find that in order to reproduce the observed $\mathrm{H} \alpha$ position-velocity diagram of the $\mathrm{HH} 444$ jet, we need a model with the following ingredients:

A quadratic, centre-to-edge velocity profile, with a maximum on-axis velocity $v_{0}=275 \mathrm{~km} \mathrm{~s}^{-1}$ and a minimum velocity $v_{0}=10 \mathrm{~km} \mathrm{~s}^{-1}$ at the edge of the jet beam. This centre-to-edge velocity profile is necessary for reproducing the observed acceleration (with increasing distances from the source) in the $\mathrm{H} \alpha$ position-velocity diagram of the $\mathrm{HH} 444$ jet.

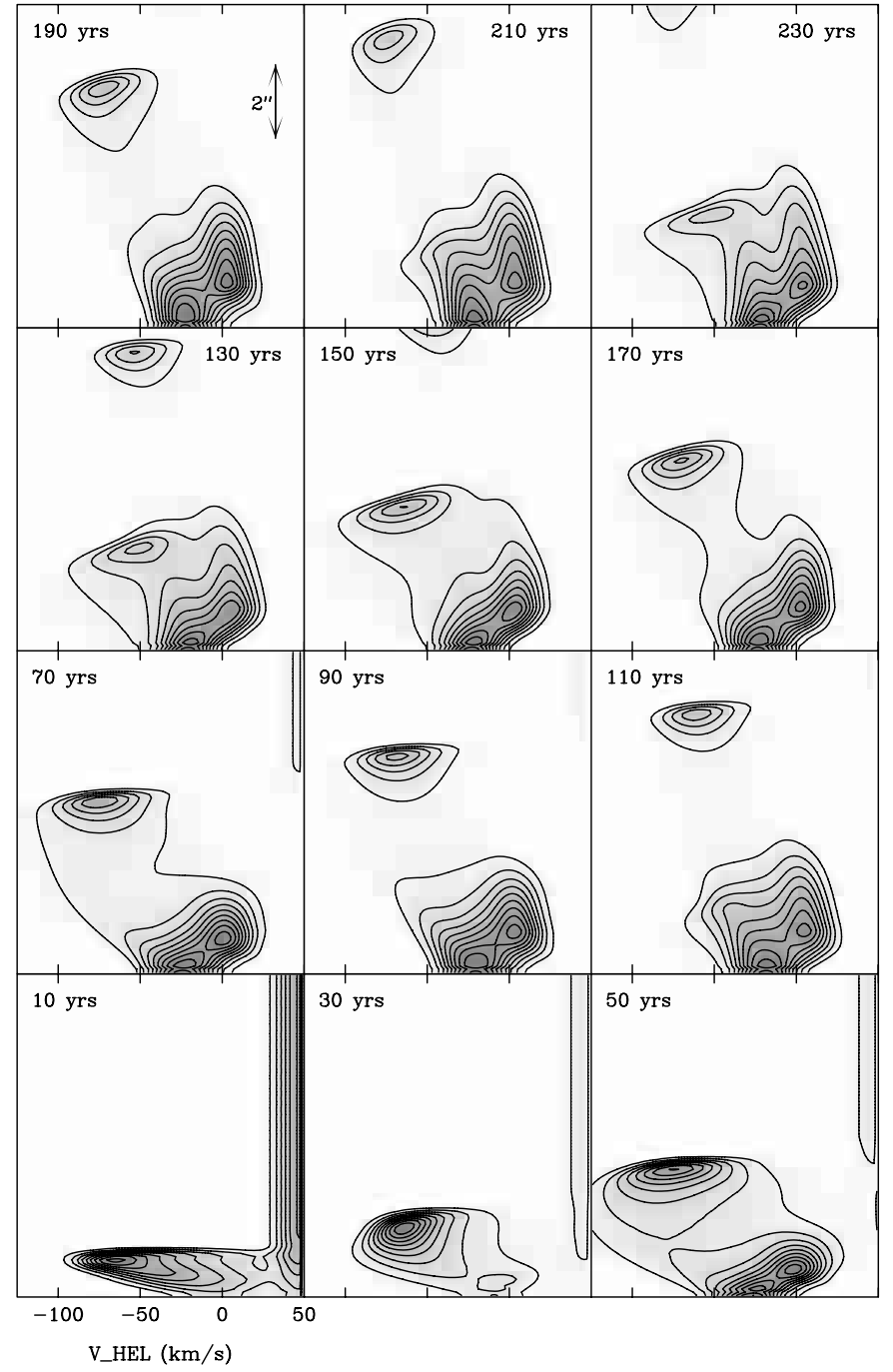

Fig. 3. Time series of the $\mathrm{H} \alpha$ position-velocity diagrams predicted from the model of $\mathrm{HH} 444$ with a centrally peaked ejection velocity cross section, a time-dependent ejection velocity and a non zero initial opening angle as described in Sect. 3

We then modulate the ejection velocity cross section with a sinusoidal time variability of the form:

$u_{0}(t)=v_{0}\left[1+0.3 \sin \left(\frac{2 \pi t}{100 \mathrm{yr}}\right)\right]$.

This velocity variability produces knots with intensities and positions similar to the ones observed along the $\mathrm{HH} 444$ jet.

We impose an initial full opening angle of $40^{\circ}$ for the jet beam. This opening angle is necessary for reproducing the observed drop in the $\mathrm{H} \alpha$ intensity with increasing distance from the source.

In Fig. 3, we show a time series of the $\mathrm{H} \alpha$ positionvelocity diagrams predicted from this model. In order to compute these diagrams, we have assumed an angle of $40^{\circ}$ (away from the observer) between the plane of the sky and the outflow axis. As the position of the spectrograph slit used in our observations is not known with enough precision, in the computed position-velocity diagrams we 
have assumed that all of the emission from the jet falls within the spectrograph slit.

From this figure, we see that at different evolutionary times we do obtain structures that resemble the $\mathrm{H} \alpha$ position-velocity diagram of HH 444 (see Fig. 2). For example, in agreement with the observations, we obtain two spatially extended components: a low velocity component, and a high velocity, accelerating component. We also obtain a third, decelerating component at distances of a few seconds of arc from the source, which corresponds to the emission from the head of the jet (at early times of the simulations) or from internal working surfaces (at later times). This component is also seen in the observations as the first knot along the $\mathrm{HH} 444$ jet.

In order to test the revelance of the different ingredients of our model, we have also computed models in which we omit some of the features described above. In Fig. 4, we compare the results from models:

b) a model in which we have set the amplitude of the velocity variability to zero. This model does produce the low and high (accelerating) velocity components, but does not have the knot observed at $\sim 5^{\prime \prime}$ from the source in $\mathrm{HH} 444$;

c) a model in which we have set the initial opening angle to zero. In this model, the resulting position-velocity diagram is completely dominated by the emission from the internal working surface, and the base of the jet is barely visible;

d) a model in which we have imposed a top-hat ejection velocity cross section. In this model, the jet emission appears at a more or less constant radial velocity as a function of position, not reproducing at all the acceleration observed in the $\mathrm{HH} 444$ position-velocity diagrams.

A comparison of these models shows that a centrally peaked ejection velocity cross section with a timedependent ejection velocity and a non-zero initial opening angle (panel a) in Fig. 4 produces the best fit to the observations of the $\mathrm{HH} 444$ jet.

Figure 5 shows the flow stratification (on the $x z$-plane) obtained for this model for integration time $t=190 \mathrm{yrs}$.

\section{Conclusions}

We have obtained $\mathrm{H} \alpha$ position-velocity diagrams of the base of the HH 444 jet, and of its main bow shock (see Reipurth et al. 1998). From the line profiles of the main bow shock, we deduce an orientation angle of $40^{\circ}$ between the outflow axis and the plane of the sky.

We also present a first comparison between predictions obtained from a 3D numerical simulation, in which the radiative transfer of the ionizing radiation from $\sigma$ Orionis is included, and the long-slit spectra of the photoionized HH 444 jet.

In the best-fit model, the jet beam is gradually unveiled by the passage of an oblique ionization front driven by the ionizing radiation from $\sigma$ Orionis. If we assume

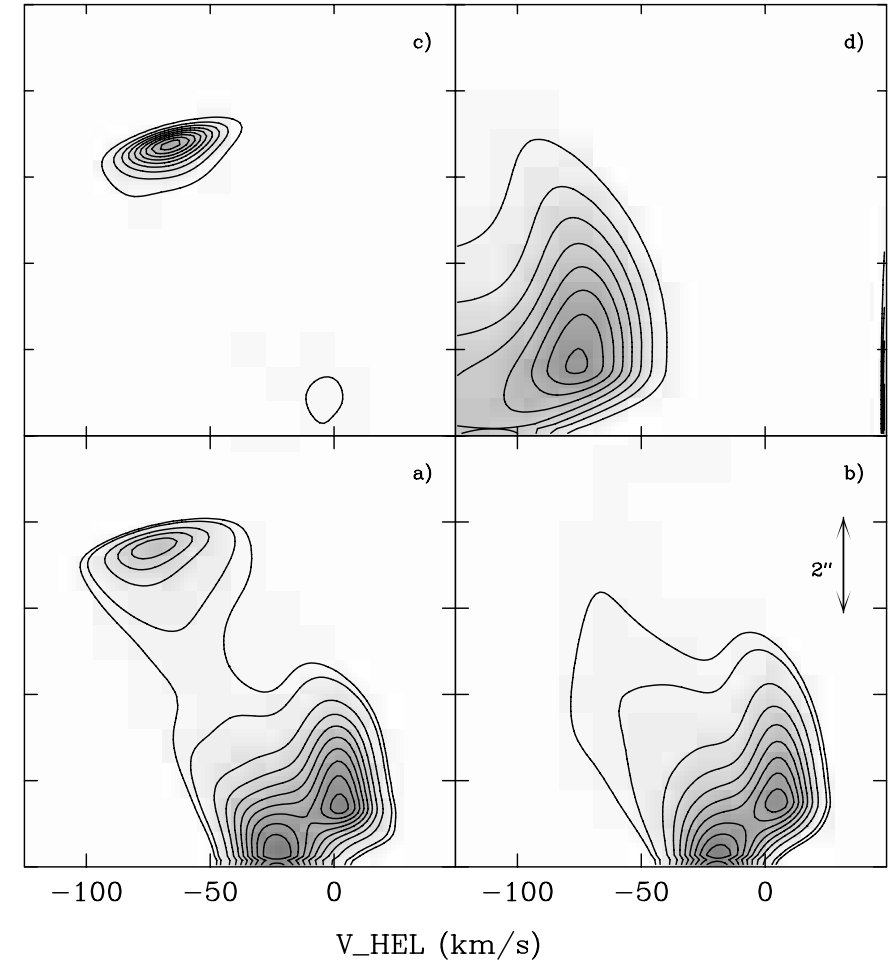

Fig. 4. Contour maps, (with lineraly spaced contours) of the $\mathrm{H} \alpha$ position-velocity map from models, a) with a centrally peaked ejection velocity cross section, a time-dependent ejection velocity and a non zero initial opening angle, b) with no velocity variability, $\mathbf{c}$ ) with a zero initial opening angle, $\mathbf{d}$ ) with a top-hat ejection velocity cross section

that the jet beam has an axially peaked velocity profile, this unveiling produces an acceleration of the emission in the predicted position-velocity diagrams, which we find is in qualitative agreement with the observed acceleration of the position-velocity diagrams of the HH 444 jet.

If we assume that the ejection velocity has a sinusoidal time variability, we produce internal working surfaces along the jet. We find that these internal working surfaces have signatures in the predicted position-velocity diagrams which resemble the line profile of the first emitting knot along the $\mathrm{HH} 444$ jet.

Finally, we find that in order to reproduce the observed decrease in the $\mathrm{H} \alpha$ intensity with increasing distances from the source, and the correct relative intensity between the base of the jet and the first emitting knot, we find that it is necessary to impose an initial opening angle of $40^{\circ}$ for the jet beam.

We should note that there is an alternative possibility to explain the observed intensity vs. distance decrease close to the base of the HH 444 jet. As we are allowing ourselves to choose an appropriate time-dependent outflow velocity in order to reproduce the structure of the HH 444 jet, we could also allow ourselves to choose an appropriate time-dependent injection density. This timedependent injection density could be chosen in such a way as to reproduce the observed $\mathrm{H} \alpha$ emission measure decrease with increasing distances from the outflow source. 


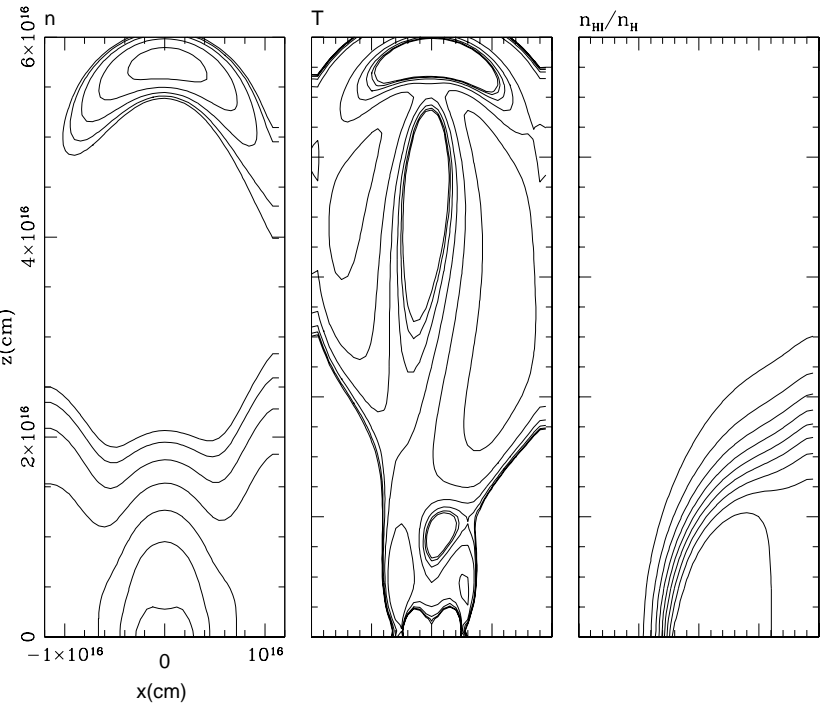

Fig. 5. Number density (of atoms and ions), temperature and neutral hydrogen fraction stratifications obtained for model the model with a centrally peaked ejection velocity cross section, a time-dependent ejection velocity and a non zero initial opening angle, (see Fig. 4a), after a $t=190$ yrs time integration. The density and tempearture stratifications are depicted with $\sqrt{2}$, logarithmic contours, and the neutral fraction is shown with linear contours in steps of 0.1

Therefore, the different elements which we have found for the HH 444 model described in the present paper have to be regarded as possibilities which do not necessarily define a unique model for this object.
Acknowledgements. L. López-Martín is in grateful receipt of a graduate scholarship from DGEP-UNAM (México). L. López-Martín and A. C. Raga acknowledge support from the CONACyT grants 27546-E and 32753-E. J. A. López acknowledges support from CONACyT and DGAPA UNAM through projects 32214-E and IN 114199, respectively. A. C. Raga acknowledges the support from a fellowship of the John Simon Guggenheim Memorial Foundation. We thank the referee, John Bally, for his insightful comments, wich have helped improve this paper.

\section{References}

Bally, J., \& Reipurt, B. 2001, ApJ, 546, 299

Bally, J., O'Dell, C. R., \& McCaughrean, M. J. 2000, AJ, 119, 2919

Hartigan, P., Raymond, J., \& Hartmann, L. 1987, ApJ, 316, 323

López-Martín, L., Raga, A. C., López, J. A., \& Meaburn, J. 2000, RMxAC, 9, 230

Meaburn, J., Blundell, B., Carling, R., et al. 1984, MNRAS, 210, 463

Raga, A. C., Mellema, G., Arthur, S. J., et al. 1999, RMxAA, 35, 123

Raga, A. C., López-Martín, L., Binette, L., et al. 2000, MNRAS, 314, 681

Raga, A. C., López-Martín, L., López, J. A., \& Meaburn, J. 2000, RMxAC, 9, 191

Reipurth, B., Bally, J., Fesen, R. A., \& Devine, D. 1998, Nature, 396, 343 